\title{
Lingual thyroid: a systematic review of hormonal suppression treatment
}

\begin{abstract}
Lingual thyroid is a rare clinical entity that is caused by the failure of the thyroid gland to descend to its normal cervical location. The pathogenesis is unclear. The occurrence of a lingual thyroid at the base of the tongue may cause various symptoms. One of the treatments for this disorder includes the use of an exogenous thyroid hormone to induce shrinkage of the gland.
\end{abstract}

Purpose: The purpose of this study was to review the effectiveness of hormone suppression treatment (HST) for the treatment of lingual thyroid.

Method: Applying established methodology, we conducted a systematic review of the literature on

the management of lingual thyroid from 1966 to December 2013.

Results: Eighty patients were treated by HST, of which 52/80 (65\%) improved. Improvement was

defined as the disappearance of the symptom that initiated HST. Of the patients, $88.3 \%$ $(68 / 77)$ were female.

Conclusion: HST was effective in about two-thirds of patients with lingual thyroid. HST treatment

should be considered a first line treatment, unless the patient presents with a life-threatening situation, such as frank bleeding or a pending airway compromise.

Keywords: lingual thyroid, ectopic thyroid, thyroid ectopia, hormone suppression treatment, hypothyroidism
Volume 2 Issue 3 - 2015

\author{
Hazim Aleid,' Amani Alharbi \\ 'Division of Otolaryngology, King Fahad Specialist Hospital, \\ Saudi Arabia \\ ${ }^{2}$ Department of Otolaryngology, Ministry of Health, Saudi Arabia
}

Correspondence: Amani Alharbi, Department of

Otolaryngology, Ministry of Health, Dammam, Saudi Arabia,

Email amani_alharbi@hotmail.com

Received: February 7, 2015 | Published: April 07, 2015

\section{Introduction}

The first case of lingual thyroid was reported following the suffocation of a newborn infant secondary to a large mass at the base of the tongue in 1869 by Hickman (per Myerson \& Smith; ${ }^{1}$ per Weider \& Parker. $^{2}$ The prevalence of a lingual thyroid has been reported to be 1 in 100,000 (Neinas, Gorman, Devine, \& Woolner). ${ }^{3}$

Autopsy studies reveal an incidence of approximately $10 \%$ in the normal population, with both sexes being equally affected (Baughman; ${ }^{4}$ Sauk). ${ }^{5}$ No geographic or social predisposition exists. The condition is more often clinically apparent in females (Kamat, Kulkarni, Desai, \& Jussawalla; ${ }^{6}$ Neinas, Gorman, Devine, \& Woolner). ${ }^{3}$

The thyroid gland develops as an endodermal bud that elongates to form the thyroglossal duct from the floor of the pharynx between the first and second branchial pouches during the third and fourth week of gestation. The duct, though it may persist, usually atrophies during the fifth week, isolating the gland from its site of origin at the foramen cecum (Myerson \& Smith). ${ }^{1}$ Failure or incomplete descent of the thyroglossal duct results in a lingual thyroid Okstad et al. ${ }^{7}$

Although an ectopic thyroid is commonly asymptomatic, glandular hypertrophy (particularly in children) of the ectopic thyroid gland may occur at any point along the descent of the primordium. The resulting locations are designated as lingual, sublingual, pretracheal (normal) and substernal. The most frequent site of an ectopic thyroid is the lingual region. In many patients, the ectopic thyroid may be the only functioning thyroid tissue (Noyek, \&Friedberg). ${ }^{8}$ An ectopic thyroid may cause dysphagia, dysphonia, bleeding or frank airway compromise.

The purpose of this study is to review the effectiveness of medical treatment, primarily hormone suppression treatment (HST).

\section{Clinically significant lingual thyroid}

The presence of lingual thyroid tissue does not preclude the presence of thyroid tissue in the neck in its normal position. However, 70 to $100 \%$ of patients with a lingual thyroid have no other functioning thyroid tissue in the neck (Neinas, Gorman, Devine, \& Woolner ${ }^{3}$ ). It has been reported that the rate of hypothyroidism is $15 \%$ and that hyperthyroidism is rarely seen (Alderson, \& Lannigan; ${ }^{9}$ Turgut et al. $)^{10}$

The complications related to ectopic thyroid are a result of its location and/or size. Pharyngeal discomfort, dysphagia or dysphonia can occur if the tumor lies in close approximation to the epiglottis. Progressive or acute airway and/or gastrointestinal tract symptoms are more frequent in children. Bleeding and ulceration are rare complications. Malignant change occurs in approximately $1 \%$ of ectopic lingual thyroids and usually results in papillary carcinoma (Diaz-Arias et al., ${ }^{11}$ Matsumoto $^{12}$ ))

The differential diagnosis of lingual thyroid includes a hypoglossal cyst, angioma, fibroma, lymphangioma or lipoma. The diagnosis of such a rare entity is relatively easy, while the treatment may still be challenging. 


\section{Materials and methods}

A systematic review of the literature was performed using established methodology for systematic reviews. The authors performed the search independently.

A Medline search of the 1966 to December 2013 database was supplemented by a search of an additional database, EMBASE (1980August, 2007). References from the papers selected for full text review were identified and reviewed.

Papers on lingual thyroid were identified using the combined, exploded medical subject heading (MeSH) terms and keywords (.mp. modifier) "Lingual Thyroid". Combining this main set with a second set of articles identified relevant studies. The resultant dataset was limited to studies in humans and those published in the English language.

\section{Inclusion criteria}

Papers were included if they reported:

Medical management with hormone suppression therapy (HST) of a lingual thyroid in any age group and in both sexes.

\section{Exclusion criteria}

a) Studies that reported other modalities as the first line of management.

b) Letters, editorials, comments and pictorial papers.

c) Studies published prior to 1966 and after 2013 .

d) Papers that reported autoimmune thyroiditis or malignant changes in the lingual thyroid.

A review of articles was performed independently by two authors
(HAE, AH). The titles of the articles were reviewed, and the titles were included if both authors agreed by general consensus to include them. When there was a disagreement, the title was included and the abstract was reviewed. The abstracts of the papers were reviewed and accepted by consensus, and when the abstracts met the inclusion criteria, the full text was obtained. Where the abstracts of accepted titles were not available, the full text of those articles were obtained and reviewed. Subsequently, the full text was reviewed to judge whether the articles met the pre-determined inclusion criteria. Information on patient demographics; presenting symptoms and clinical profiles; relevant investigations (notably thyroid function tests (T3, T4 and TSH)); and other diagnostic tests, including ultrasonography (USG), magnetic resonance imaging (MRI) and radio iodine uptake, were abstracted. Information regarding the management of patients with lingual thyroid was extracted from the relevant articles by two authors independently (HAE, AH). Any discrepancies were resolved by mutual consensus.

Data were analyzed using descriptive statistics only given the nature of the information that was abstracted.

\section{Results and discussion}

Two-hundred twenty-eight titles of articles were reviewed. Onehundred sixty seven abstracts of the papers that had accepted titles were reviewed. Sixty five abstracts were excluded, as no medical treatment was discussed, and 102 full text articles were selected. Subsequently, 54 full text articles met our inclusion criteria. Forty eight articles were excluded (the list will be provided upon request from the corresponding author). Management of lingual thyroid exclusively by surgical excision was reported in 39\% (19/48) of the studies. Other reasons for exclusion were: no medical treatment initiated because of minor symptoms (10articles) or no cases discussed or patient condition mentioned (17articles). Two papers reported treatment with radioiodine 131 (Figure 1).

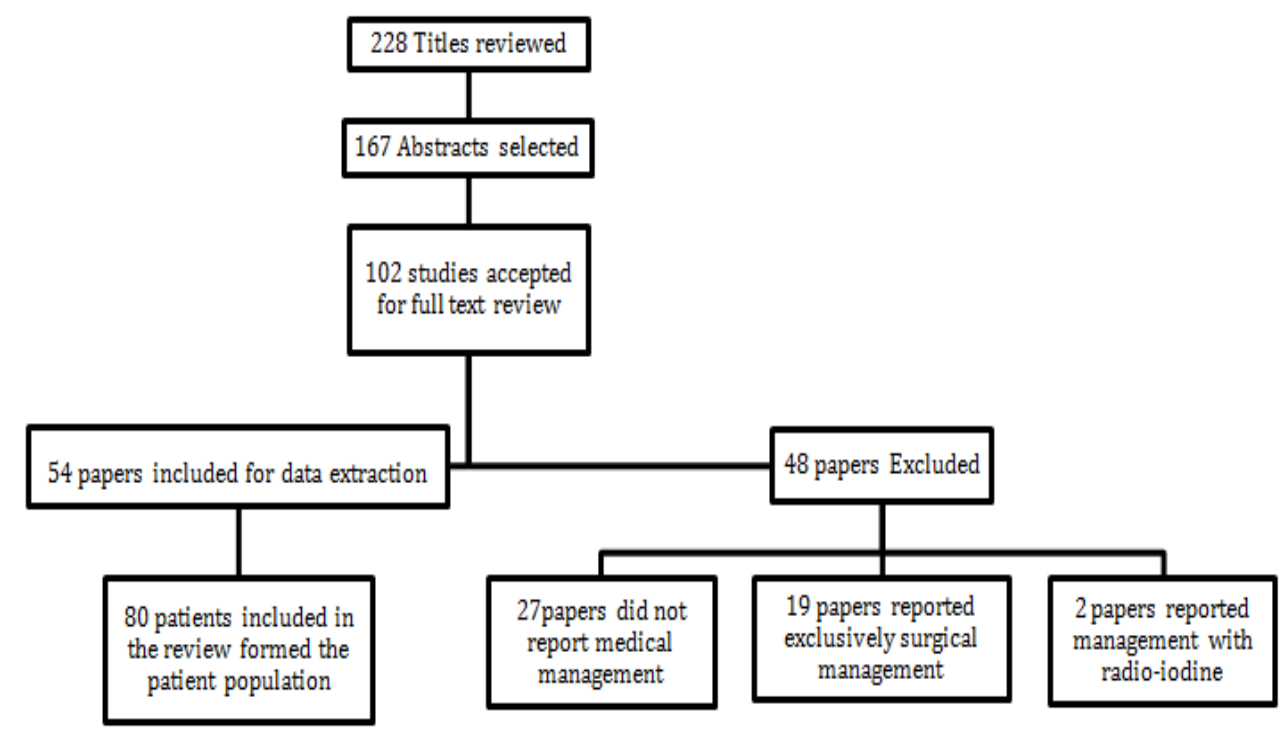

Figure I Flow chart of the literature review. 
The full text of 54 articles was reviewed for data extraction (Appendix1) (Appendix2). A total of 80 patients received medical management for their symptoms, while 2 patients were asymptomatic when they received treatment. Medical management was in the form of hormonal suppression therapy (HST).

Where information on age was available, the ages ranged from 3 months to 83years. There was no information on the ages of nine patients (Kamat, Kulkarni, Desai, \& Jussawalla, 1979, ${ }^{6}$ (Prasad \& Bhat, 2000)). ${ }^{13}$ Forty four of the 73 patients $(60 \%)$ were below the age of 18 years. One study reported a case series of 12 patients in which 5 patients met our inclusion criteria; one patient was lost to follow up, and the remaining six underwent an operation for their condition. From the information on the age of the cases, it was apparent that patients often report for treatment in the second decade of life (Kamat, Kulkarni, Desai, \& Jussawalla, 1979). ${ }^{6}$ In our review, the majority of patients presented in the first and second decade of life (Figure 2).

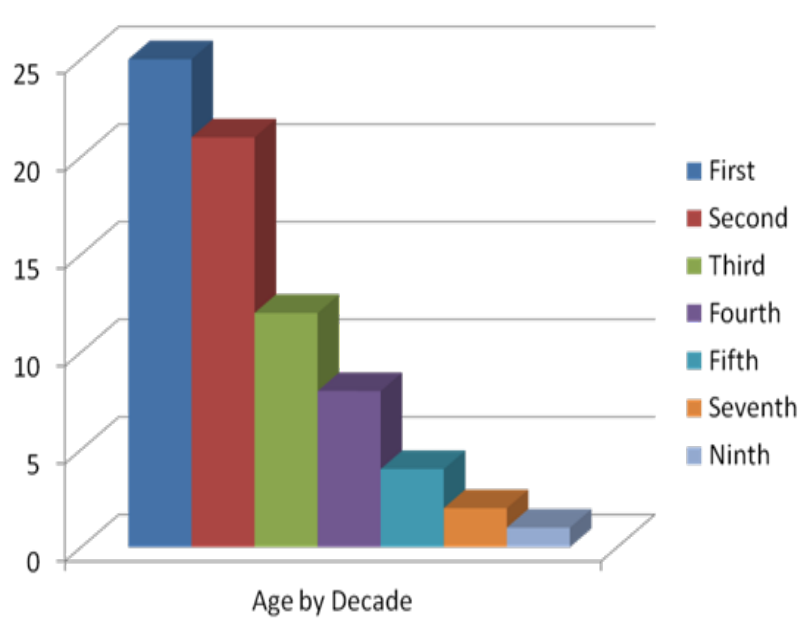

Figure 2 Distribution of Age by Decade in the Study Population.

Where gender was reported, females accounted for $88.3 \%(68 / 77)$ of the patient population reviewed.

Patients presented with symptoms that were either related to the size of the gland or to the ectopic placement of the gland. There was heterogeneity in the reported sizes of the lingual thyroid, with the biggest reported size being $5 \mathrm{~cm} \times 4 \mathrm{~cm}$ and the smallest $1 \mathrm{~cm} \times 0.5 \mathrm{~cm}$ The most common presenting symptoms were dysphagia reported by $48.7 \%$ of patients $(40 / 82)$, followed by foreign body sensation in the throat in $21.9 \%(18 / 82)$. In 21 patients, dysphagia was the only symptom. In thirteen patients, the ectopic thyroid was an incidental finding of an asymptomatic lump. Bleeding was a rare symptom reported by only three patients (Table 1). Several patients presented with multiple symptoms.

HST is based on the concept that with an exogenous hormone the endocrine gland will decrease in size. With ectopic thyroid tissue, specifically a lingual thyroid, the protocol for treatment, including the dose, time and length of treatment, is unclear in the literature. The effectiveness of treatment to eliminate the symptoms and the length of treatment before moving on to another treatment modality is also unknown. The dose of HST was reported for 53 patients and varied depending on the age of the patient and the status of the thyroid function at presentation.

Although the choice of treatment depends on several factors, HST was effective in 49 patients $(61.2 \%)$ in a slow but significant way. The clinical management of lingual thyroid remains somewhat controversial. The use of suppressive therapy with an exogenous thyroid hormone is the mainstay of medical management. Clinical examination can then be performed and thyroid function monitored at regular intervals. Frequent follow-up and examination may be necessary during periods of metabolic stress. Surgical intervention is necessary for selected patients who become symptomatic or have a worsening of their initial symptoms while on suppressive therapy.

Table I Presenting symptomatology

\begin{tabular}{ll}
\hline Presenting symptom & $\begin{array}{l}\text { Number of } \\
\text { Patients N=82 }\end{array}$ \\
\hline Coughing & $5(6 \%)$ \\
Bleeding & $3(3.6 \%)$ \\
Dysphagia & $40(48.7 \%)$ \\
Dysphonia & $6(7.3 \%)$ \\
Dyspnea \& respiratory symptoms & $17(20.7 \%)$ \\
Foreign body sensation in the throat & $18(21.9 \%)$ \\
Sore throat & $8(9.7 \%)$ \\
Asymptomatic lump & $13(15.8 \%)$
\end{tabular}

*Percentages add up to greater than $100 \%$, as some patients presented with more than one symptom

Improvement was defined as the disappearance of the reason that initiated the hormonal suppression therapy. Initial improvement was achieved in 52/80 patients $(65 \%)$. However, three patients stopped responding to HST after a period of time and underwent another modality of treatment. There were $28 / 80(35 \%)$ of patients who had no or partial improvement of their presenting symptom or symptoms (Table 2). Twenty five patients whose symptoms improved were below 18 years of age, while 19 were above 18, and no age was given in 5 patients. Fifteen and ten patients who reported no improvement were under 18 and above 18 years old, respectively. A total of 31/80 $(38.75 \%)$ had another modality of treatment or were lost to follow up (Table 2).

Of the patients who improved, 65.3\% (32/49) patients were in a hypothyroid or compensated hypothyroid status at the start of treatment, while six were euthyroid (12.2\%), and the hormonal status was unknown in eleven patients. In the group that had no improvement (31/80 or $38.7 \%), 12$ patients were in a hypothyroid or compensated hypothyroid status (38.7\%), 13 were euthyroid (41.9\%) and six were of unknown hormonal status prior to treatment commencing.

The duration of HST in patients who showed no improvement (26/80 patients) varied between one month (Barnes, Olsen, \& Morgenthaler, 2004 ${ }^{14}$ ) and 8years (Zitsman, Lala, \& Rao, ${ }^{15}$ ) with an overall mean of 22months. Montgomerry in 1935 reported a high rate of lingual thyroid among females compared to males. Our systematic review found similar high rates $(88.3 \%)$ among females. The frequency of lingual thyroid was higher in the first 3 decades of life (Figure 2). 
Table 2 Management of Lingual Thyroid patients.

\begin{tabular}{ll}
$\begin{array}{l}\text { Final management of those who } \\
\text { initially improved with HST }\end{array}$ & $\begin{array}{l}\text { Number of } \\
\text { Patients } \mathbf{N}=\mathbf{5 2}\end{array}$ \\
\hline $\begin{array}{l}\text { Maintained on HST } \\
\text { Lost to follow up }\end{array}$ & I \\
Received surgical excision & \\
$\qquad \quad$ One for non compliance \\
$\quad \begin{array}{l}\text { One for a gradual increase } \\
\text { in size }\end{array}$
\end{tabular}

Initial Improvement Occurred 52/80 (65\%)

\begin{tabular}{ll}
\hline $\begin{array}{l}\text { Final management of those } \\
\text { who did not have any initial } \\
\text { improvement with HST }\end{array}$ & $\begin{array}{l}\text { Number of } \\
\text { Patients N=26 }\end{array}$ \\
\hline Maintained on HST & 3 \\
I I3I & 2 \\
CO2 Laser ablation & 3 \\
Radiofrequency ablation & $\mathrm{I}$ \\
Surgery & $\mathrm{I}$ \\
\hline
\end{tabular}

No Improvement with Initial Management $\quad 26 / 80$ (32.5\%)

\begin{tabular}{ll}
\hline $\begin{array}{l}\text { Final management of those who } \\
\text { had partial remission of their } \\
\text { symptoms with HST }\end{array}$ & $\begin{array}{l}\text { Number of } \\
\text { Patients } \mathbf{N}=\mathbf{2}\end{array}$ \\
\hline II3I & $\mathrm{I}$ \\
CO2 Laser ablation & $\mathrm{I}$
\end{tabular}

Partial Remission 2/80 (2.5\%)

\section{Conclusion}

There is no consensus in the literature for the correct management of lingual thyroid. However, HST should be attempted first if life threatening symptoms are absent. The response should be monitored by following thyroid hormone markers (T4, T3, TSH and Thyroglobulin) and the patient's clinical status. There is an approximately $61 \%$ chance that the symptoms will improve significantly. With no known protocol, the dose and duration of the treatment should be customized for each individual patient. Close collaboration between the endocrinologist and surgeon is paramount.

\section{Acknowledgments}

None.

\section{Conflicts of interest}

The authors declare that there are no conflicts of interest.

\section{Funding}

None.

\section{References}

1. Myerson M, Smith HW. Lingual thyroid--a review. Conn Med. 1966;30(5):341-344.

2. Weider DJ, Parker W. Lingual thyroid: Review, case reports, and therapeutic guidelines. Ann Otol Rhinol Laryngol. 1977;86(6 Pt 1):841-848.

3. Neinas FW, Gorman CA, Devine KD, et al. Lingual thyroid. Clinical characteristics of 15 cases. Ann Intern Med. 1973;79(2):205-210.

4. Baughman RA. Lingual thyroid and thyroglossal tract remnant a clinical and histopathologic study review of the literature. Oral Surg Oral Med Oral Pathol. 1972;34(5):781-799.

5. Sauk JJ Jr. Ectopic lingual thyroid. J Pathol. 1970;102(4):239-243.

6. Kamat MR, Kulkarni JN, Desai PB, et al. Lingual thyroid: A review of 12 cases. Br J Surg. 1979;66(8):537-539.

7. Okstad S, Mair JW, Sunddsfjord JA, et al. Ectopic thyroid tissue in head and neck. J Otolaryngol. 1986;15(1):52-55.

8. Noyek AM, Friedberg J. Thyroglossal duct and ectopic thyroid disorders. Otolaryngol Clin North Am. 1981;14(1):187-201.

9. Alderson DJ, Lannigan PC. Lingual thyrois presenting after previous thyroglossal cyst excision. J Laryngol Otol. 1994;108(4): 341-343.

10. Turgut S, Murat Ozcan K, Celikkanat S, et al. Diagnosis and treatment of lingual thyroid: A review. Rev Laryngol Otol Rhinol. 1997;118(3):189-192.

11. Diaz-Arias AA, Bickel JT, Loy TS, et al. Follicular carcinoma with clear cell change arising in lingual thyroid. Oral Surg Oral Med Oral Pathol. 1992;74(2):206-211.

12. Matsumoto K, Watanabe Y, Asano G. Thyroid papillary carcinoma arising in ectopic thyroid tissue within a branchial cleft cyst. Pathol Int. 1999;49:444-446.

13. Prasad KC, Bhat KV. Surgical management of lingual thyroid: A report of four cases. J Oral Maxillofac Surg. 2000;58(2):223-227.

14. Barnes TW, Olsen KD, Morgenthaler TI. Obstructive lingual thyroid causing sleep apnea: A case report and review of the literature. Sleep Med. 2004;5(6):605-607.

15. Zitsman JL, Lala VR, Rao PM. Combined cervical and intraoral approach to lingual thyroid: A case report. Head Neck. 1998;20(1):79-82. 